\title{
\begin{tabular}{l|l} 
Mitraries & DSpace@MIT
\end{tabular}
}

\author{
MIT Open Access Articles
}

\section{Distributed Throughput Maximization in Wireless Networks via Random Power Allocation}

The MIT Faculty has made this article openly available. Please share how this access benefits you. Your story matters.

Citation: Hyang-Won Lee, E. Modiano, and Long Bao Le. “Distributed throughput maximization in wireless networks via random power allocation." Modeling and Optimization in Mobile, Ad Hoc, and Wireless Networks, 2009. WiOPT 2009. 7th International Symposium on. 2009. 1-9. () 2009 Institute of Electrical and Electronics Engineers.

As Published: http://dx.doi.org/10.1109/WIOPT.2009.5291606

Publisher: Institute of Electrical and Electronics Engineers

Persistent URL: http://hdl.handle.net/1721.1/59825

Version: Final published version: final published article, as it appeared in a journal, conference proceedings, or other formally published context

Terms of Use: Article is made available in accordance with the publisher's policy and may be subject to US copyright law. Please refer to the publisher's site for terms of use. 


\title{
Distributed Throughput Maximization in Wireless Networks via Random Power Allocation
}

\author{
Hyang-Won Lee, Eytan Modiano and Long Bao Le \\ Massachusetts Institute of Technology, Cambridge, MA 02139 \\ Email: \{hwlee, modiano, longble\}@mit.edu
}

\begin{abstract}
We consider throughput-optimal power allocation in multi-hop wireless networks. The study of this problem has been limited due to the non-convexity of the underlying optimization problems, that prohibits an efficient solution even in a centralized setting. We take a randomization approach to deal with this difficulty. To this end, we generalize the randomization framework originally proposed for input queued switches to an SINR rate-based interference model. Further, we develop distributed power allocation and comparison algorithms that satisfy these conditions, thereby achieving (nearly) $100 \%$ throughput. We illustrate the performance of our proposed power allocation solution through numerical investigation and present several extensions for the considered problem.
\end{abstract}

Index Terms-Power allocation, wireless scheduling, capacity region, graph-based interference model, SINR interference model.

\section{INTRODUCTION}

Resource allocation in multihop wireless networks involves solving a joint link scheduling and power allocation problem which is very difficult in general [1], [2]. Due to this difficulty, most of the existing works in the literature consider a simple setting where all nodes in the network use fixed transmission power levels where the resource allocation problem degenerates into simply a link scheduling problem [3]-[6]. Furthermore, the link scheduling problem has been mostly performed assuming a simplistic graph-based interference model.

In fact, the resource allocation problem has been mainly considered in two different network settings in the literature. The first setting is a static one which does not take randomness in traffic arrival processes into consideration. In particular, it is usually assumed users either always have unlimited amount of traffic to transmit or have predetermined traffic demands. Here, resource allocation aims at performing fair radio resource allocation among competing traffic flows or developing resource allocation algorithms which have nice performance properties (e.g., constructing minimum length schedule to support a predetermined traffic demands) [7]-[10], [11]. The second setting assumes random arrival traffic and one of the main objectives of the resource allocation problem is to maximize the average arrival rates which can be supported while maintaining stability of network queues.

This research was supported by National Science Foundation under ITR Grant CCR-0325401 and ARO Muri grant number W911NF-08-1-0238. Hyang-Won Lee was partially supported by the Korea Research Foundation Grant funded by the Korean Government(MOEHRD)(KRF-2007-357D00164). Long Bao Le was partially supported by NSERC Postdoctoral Fellowship.
In the seminal work of [12], Tassiulas and Ephremides introduce the concept of stability region, defined as the set of all arrival rate vectors that can be stably supported. They also propose a joint routing and scheduling policy that achieves $100 \%$ throughput, meaning that it stabilizes the network whenever the arrival rate vector is in the stability region. More recently, this throughput-optimal policy has been extended to wireless networks with power control [13], [14] and for the scenario where arrival rates lie outside the capacity region [15].

All these resource allocation algorithms, however, require repeatedly solving a global optimization problem which is NP-hard in general [16], [2]. Hence, in multi-hop wireless networks, it may be impractical to find its solution in every time slot due to limited computation capability, and the need for distributed operation. As an alternative, distributed greedy scheduling has been proposed and analyzed [6], [16]-[19]. However, most of the existing works in this context adopt the graph-based interference models, where transmissions on any two links in the network are assumed to be either conflict or conflict-free. Moreover, the use of greedy scheduling typically results in throughput reduction of at least $50 \%$.

It has been recognized that graph-based interference models may be overly simplistic because they ignore the cumulative effect of wireless interference. However, going beyond these simplistic interference models is challenging. In fact, the power allocation problem under the SINR rate-based interference model is non-convex; therefore, obtaining a global optimal power allocation even in a centralized manner is not practical. This non-convexity issue in the power allocation problem has been addressed by several papers [7], [9] considering either the high or low SINR regimes.

This paper deals with the throughput-optimal power allocation problem under the SINR rate-based interference model. We take a randomization approach to circumvent the difficulty of the problem. The randomization technique was originally developed for input queued switches [20], and later extended for multi-hop wireless networks assuming the graph-based primary and secondary interference models [3], [4]. Its key feature is that it does not seek to find an optimal schedule in every time slot, and consequently, solving a difficult scheduling problem can be avoided. Motivated by this observation, our work attempts to alleviate the difficulty in solving the non-convex optimization problem involved in optimal power allocation, using randomization.Our contributions can be summarized as follows: 
- We generalize the randomization framework to the SINR rate-based interference model, so that we can deal with the power allocation problem using randomization technique.

- We develop a new optimality condition for random power allocation that enables the application of the randomization framework to power allocation problem, and develop a randomized power allocation that satisfies the new optimality condition.

- We develop a distributed gossip-based comparison mechanism together with the randomized power allocation that achieves $100 \%$ throughput.

The rest of the paper is organized as follows. In Section II, we present the system model and describe the problem under consideration. In Section III, we review the randomized scheduling framework and discuss how to extend this framework to wireless networks with power control assuming SINR rate-based interference model in section IV. In section V, we present implementation for the comparison step of our newlyproposed power allocation framework by using a randomized gossip algorithm. Simulation results are presented in section VI and conclusions are stated in section VII.

\section{Model AND PROBlem Description}

We consider a multi-hop wireless network modeled by a graph $G=(V, E)$, where $V$ is the set of nodes and $E$ is the set of links. Let $N$ be the number of nodes, i.e., $N=|V|$. It is assumed that there is a link between two neighboring nodes if they want to communicate with each other.We assume that time is slotted and a time slot interval is of unit length. Let $V(a)$ be the set of node $a$ 's neighbors, i.e., $V(a)=\{b \in V$ : $(a, b) \in E\}$. We assume bidirectional links, hence link $(a, b)$ exists whenever $(b, a)$ does. For simplicity of exposition, we assume that there is only single-hop traffic and single channel available in the network. Node $a$ maintains a data buffer for each outgoing link $(a, b)$, and its backlog at time $t$ is denoted by $q_{a b}(t)$.

Denote by $p_{a b}$ the transmit power allocated to link $(a, b)$. Each node $a$ has a limited power budget $P_{a}^{\max }$, and the total transmit power constraint can be written as $\sum_{b \in V(a)} p_{a b} \leq$ $P_{a}^{\max }$. We assume SINR rate-based interference model. That is, under a power allocation vector $p=\left[p_{a b}, \forall(a, b) \in E\right]$, link $(a, b)$ 's rate $r_{a b}(p)$ is given by

$$
\log \left(1+\frac{g_{a b} p_{a b}}{n_{b}+\sum_{i \in V(a) \backslash\{b\}} g_{a b} p_{a i}+\sum_{i \neq a} g_{i b} \sum_{j \in V(i)} p_{i j}}\right),
$$

where $n_{b}$ is the noise power, and $g_{a b}$ is the channel gain from node $a$ to $b$. It is assumed $g_{a b}=\infty$ if $a=b$. Since the nodes are static, the channel gains are assumed to be fixed over time. Note that the second term in the denominator of (1) is self-interference, and the third is mutual interference.

Let $A_{a b}(t)$ represent the amount of exogenous data that arrive to the buffer at the source of link $(a, b)$ during slot $t$, and $p(t)$ the power allocation vector for slot $t$. Then, the backlog $q_{a b}(t)$ evolves according to the following dynamics:

$$
q_{a b}(t+1)=\max \left[0, q_{a b}(t)-r_{a b}(p(t))\right]+A_{a b}(t) .
$$

The arrival process $A_{a b}(t)$ is assumed to be i.i.d. over time with average $\lambda_{a b}$, i.e., $E\left[A_{a b}(t)\right]=\lambda_{a b}, \forall t$. We assume that all arrival processes $A_{a b}(t)$ have bounded second moments and they are upper-bounded by $A_{\max }$ (i.e., $A_{a b}(t) \leq A_{\max }$, $\forall(a, b) \in E)$. Now, we define the network stability and characterize the stabilized arrival rates. if

Definition 1: A queue $q_{a b}(t)$ is called to be strongly stable

$$
\limsup _{t \rightarrow \infty} \frac{1}{t} \sum_{\tau=0}^{t-1} E\left\{q_{a b}(\tau)\right\}<\infty .
$$

A network of queues is called strongly stable if all individual queues are strongly stable.

Let us drop the indices of a variable to denote its vector form, for example, $q(t)=\left[q_{a b}(t), \forall(a, b) \in E\right]$. Define capacity region denoted as $\Lambda$ as the union of arrival rate vectors $\lambda=\left(\lambda_{a b},(a, b) \in E\right)$ such that there exists a scheduling policy which stabilizes the network queues. In [13], the capacity region for wireless networks with power control was characterized. Specifically, capacity region $\Lambda$ consists of all arrival rate vectors $\lambda=\left(\lambda_{a b},(a, b) \in E\right)$ such that there exists a power vector $p$ in a feasible region of transmit power vector, defined by $F=\left\{p \geq 0: \sum_{b \in V(a)} p_{a b} \leq P_{a}^{\max }, \forall a \in V\right\}$ such that

$$
\lambda_{a b} \leq r_{a b}(p), \forall(a, b) \in E .
$$

In addition, the throughput-optimal power allocation vector solves the following "max-weight" problem in each time slot

$$
p^{*}(t)=\arg \max _{p \in F} \sum_{(a, b)} q_{a b}(t) r_{a b}(p),
$$

Note that in the graph-based interference model, link rates are fixed and the resource allocation problem degenerates into the link scheduling problem. Here, the max-weight scheduling policy which returns a feasible schedule achieving the maximum weight in each time slot is throughput-optimal.

In [14] and [13], it was shown that the power allocation policy solving the above problem for every slot $t$ achieves throughput optimality, meaning that it stabilizes the network whenever the arrival rate vector is within the stability region. However, the optimization problem (5) is nonconvex in $p$, and hence, it may not be possible to find an optimal power vector for every time slot $t$, even in a centralized manner. We address this issue by using randomization [3], [4], [20] and gossiping [21], originally proposed for input queued switches and wireless networks under graph-based interference models.

\section{RANDOMIZATION FRAMEWORK}

\section{A. Background on Randomization Framework}

The randomization approach was first developed for scheduling in input queued switches [20], and extended for 


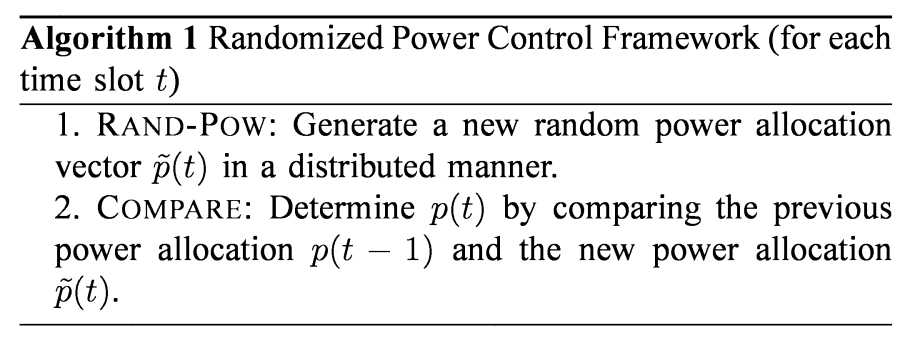

distributed operations in multi-hop wireless networks [3], [4]. Recall that under these settings, a feasible schedule is to be found in each time slot. The key feature of the randomization approach is that it does not seek to find an optimal schedule in every slot, and hence, it can significantly reduce the computation overhead. In every time slot, the randomization framework does the following:

(i) RAND-SCH: generate a new random schedule,

(ii) COMPARE: decide on the current schedule by comparing and selecting the better of the new and old schedules.

Lemma 1 ( [20]): Under the condition that the newly generated schedule in RAND-SCH is optimal with positive probability, the randomization framework achieves $100 \%$ throughput.

Note that in an input queued switch the number of possible activations is finite. Hence, it is trivial to develop a random algorithm to satisfy the condition in Lemma 1 . Moreover, the comparison in a switch can be done in a centralized manner. However, in multi-hop wireless networks, the COMPARE step is challenging because each node must compare the networkwide weighted sum rates achieved by the two schedules in a distributed manner. In [3], this comparison is localized over connected subgraphs consisting of old and new link activations; where the decisions in one subgraph do not affect the decisions at other subgraphs. The communication overhead can be substantially reduced using this localization.

\section{B. Extension to SINR Rate-Based Interference Model}

Our work in this paper is motivated by the intuition that the difficulty due to the non-convexity in (5) can also be alleviated using this randomization technique. A natural extension of the randomization framework to SINR rate-based interference model is shown in Algorithm 1. The key challenge in this setting is that it may not be possible to devise a power allocation policy RAND-POW that has a positive probability of being optimal since the optimal power allocation takes on real-values. Consequently, the randomization approach to the power allocation problem will not be able to achieve $100 \%$ throughput as in the case of the graph-based interference model. We address this issue by generalizing the condition on RAND-SCH in the graph-based interference model; namely, the newly generated power vector is not required to be optimal, but is required to be within a small factor of optimal.

Another challenge lies in the COMPARE part, as the localized comparison in the graph-based interference model may not work in our setting. With the SINR rate-based interference model, the interference level experienced at a node is affected by all the other nodes in the network. Hence, the localized comparison may lead to a wrong decision, and a networkwide comparison will be inevitable. To resolve this problem, we will use randomized gossiping [21]. Note that the objective value in (5) can be written as $q(t)^{T} r(p)$. The following is the generalized condition on RAND-POW.

Condition 1 (C1): For every time slot $t$,

$$
\operatorname{Pr}\left[q(t)^{T} r(\tilde{p}(t)) \geq\left(1-\gamma_{1}\right) q(t)^{T} r\left(p^{*}(t)\right)\right] \geq \delta_{1}>0,
$$

where $\gamma_{1}$ and $\delta_{1}$ are some positive constants.

Condition $C 1$ allows for the possibility that the new power allocation is within a factor of optimal. Notice that when $\gamma_{1}=0, C 1$ becomes the condition on RAND-SCH in [3], [20] which requires the new scheduling to be optimal with positive probability. This generalization is the key to dealing with the power control problem (5) using the randomization approach, and the optimality loss under this condition will be characterized, in Theorem 1.

The following is the condition on COMPARE adopted from [3].

Condition $2(C 2,[3])$ : For every time slot $t$, it is satisfied $q(t)^{T} r(p(t)) \geq\left(1-\gamma_{2}\right) \max \left\{q(t)^{T} r(p(t-1)), q(t)^{T} r(\tilde{p}(t))\right\}$ with probability at least $1-\delta_{2}$, where $\gamma_{2}$ and $\delta_{2}\left(\ll \delta_{1}\right)$ are some positive constants.

Condition $C 2$ requires that the weight attained by the chosen power vector $p(t)$ should not be less than some factor of the maximum of the weights obtained by $\tilde{p}(t)$ and $p(t-1)$. This condition was considered in [3] to account for imperfect comparison in multi-hop networks.

Now, the achievable stability region under our randomization framework can be characterized as follows:

Theorem 1: If RAND-POW and COMPARE in Algorithm 1 satisfy $C 1$ and $C 2$, then it stabilizes the network for any arrival rate vector in $\rho \Lambda$ where $\rho<1-\left(\gamma_{1}+\left(1-\gamma_{1}\right) \gamma_{2}\right)-2 \sqrt{\frac{\delta_{2}}{\delta_{1}}}$. Proof: See the Appendix.

When $\gamma_{1}$ is 0 , i.e., when a new power vector is optimal with probability $\delta_{1}$, the obtained throughput mainly depends on the comparison performance $\left(\gamma_{2}\right)$. However, the throughput loss increases as $\gamma_{1}$ increases. In case of perfect comparison (i.e., $\gamma_{2}=0$ and $\delta_{2}=0$ ), the throughput loss depends only on the optimality loss in the random power allocation. In brief, our randomized power control framework can achieve nearly $100 \%$ throughput if we can develop a power allocation policy (RAND-POW) and a comparison algorithm (COMPARE) satisfying conditions $C 1$ and $C 2$ with small $\gamma_{1}, \gamma_{2}$ and $\delta_{2}$. In the rest of the paper, we focus on developing such algorithms.

\section{Implementation}

For better presentation, we illustrate the structure of time frame in our randomization framework. As shown in Figure 1 , a time slot consists of 4 parts including pick, training, comparison and data transmission. In the pick slot, a new power allocation is randomly generated, i.e., the transmitterreceiver pairs are selected and also transmit power levels are selected as well. The training slot consists of three mini slots, 


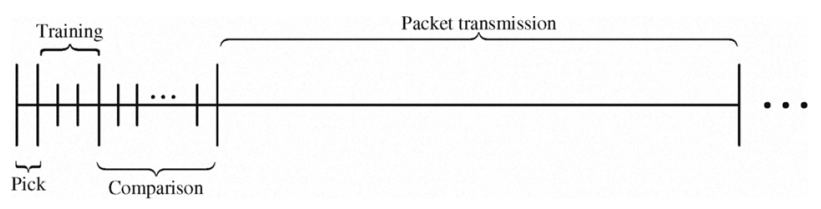

Fig. 1. Time slots in randomization framework

and it is used for computing the weighted sum rates under the old power allocation and the new power allocation. Once every node obtains these values, they compare the old value and the new value by running a distributed gossiping in the comparison slot, and select the better power allocation. The data is finally transmitted using the selected power allocation. We will discuss the detail of each part.

\section{RANDomized Power Allocation}

We present a power allocation policy RAND-POW that satisfies $C 1$, i.e., finds with positive probability a power vector within a small factor of the optimal value in (5). The problem (5) is to maximize

$$
\begin{aligned}
p^{*}=\arg \max _{p \in F} \sum_{a \in V} \sum_{b \in V(a)} q_{a b} \times \\
\log \left(1+\frac{g_{a b} p_{a b}}{n_{b}+g_{a b} \sum_{i \in V(a) \backslash b} p_{a i}+\sum_{i \neq a} g_{i b} \sum_{j \in V(i)} p_{i j}}\right),
\end{aligned}
$$

where $F=\left\{p \geq 0: \sum_{b \in V(a)} p_{a b} \leq P_{a}^{\max }, \forall a \in V\right\}$. Clearly, the new power vector $\tilde{p}$ in RAND-POW is desired to be as close to $p^{*}$ as possible, and hence, identifying the optimality properties of (7) would be helpful for generating such $\tilde{p}$. The following lemma characterizes some useful properties of $p^{*}$.

Lemma 2: Under the optimal power allocation $p^{*}$ in (7),

(i) A node transmits to at most one of its neighbors,

(ii) A node does not transmit while receiving, and vice versa.

Proof: Recall the assumption $g_{a a}=\infty, \forall a$. Under this assumption, if a node transmits to a transmitting node, it will achieve zero rate due to infinite interference. Hence, at optimal $p^{*}$, case (ii) does not happen.

To prove (i), let $p_{a}^{*}=\sum_{b \in V(a)} p_{a b}^{*}$, i.e., $p_{a}^{*}$ is the total power transmitted by node $a$ at optimal point. It is obvious that solving the problem (7) with additional constraints $\sum_{b \in V(a)} p_{a b}=p_{a}^{*}, \forall a$ will obtain the same optimal solution. In this case, the objective function in (7) can be written as

$$
\sum_{a} \sum_{b \in V(a)} q_{a b} \log \left(1+\frac{g_{a b} p_{a b}}{n_{b}+g_{a b}\left(p_{a}^{*}-p_{a b}\right)+\sum_{i \neq a} g_{i b} p_{i}^{*}}\right) \text {. }
$$

Clearly, changing transmit power $p_{a b}, \forall b \in V(a)$ under fixed total power does not affect mutual interference, but only change self-interference. Hence, the new optimization problem can be solved separately with respect to each node, i.e., for each $a$, we only need to maximize

$$
\sum_{b \in V(a)} q_{a b} \log \left(1+\frac{g_{a b} p_{a b}}{n_{b}+g_{a b}\left(p_{a}^{*}-p_{a b}\right)+\sum_{i \neq a} g_{i b} p_{i}^{*}}\right)
$$

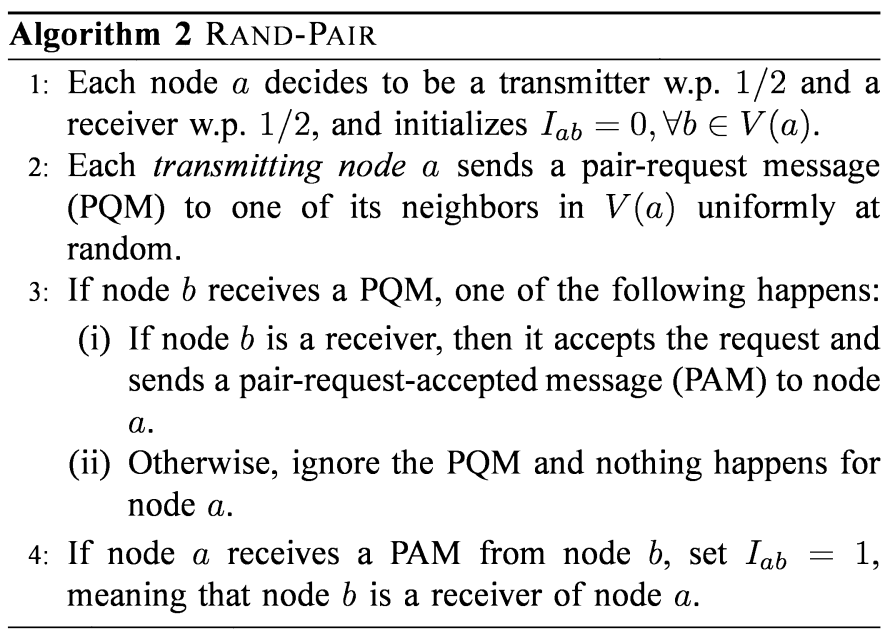

subject to $p \geq 0$ and $\sum_{b \in V(a)} p_{a b}=p_{a}^{*}$. Since this function is strictly convex in $\left[p_{a b}, \forall b \in V(a)\right]$, it is maximized at corner point, i.e., $p_{a b}=p_{a}^{*}$ for some $b \in V(a)$ and zero for all others. This shows that it is optimal to transmit to at most one node.

According to Lemma 2, at the optimal point, a node is not allowed to transmit to multiple neighbors, and to be a transmitter and receiver simultaneously. Note, however, that it is possible for a node to receive from multiple transmitters, and this should be discriminated from a matching in which a node cannot be shared by multiple edges. For ease of exposition, a definition of pairing is introduced as follows:

Definition 2: Assume that the head and the tail of a directed edge denote a transmitter and a receiver respectively. A directed subgraph of $G$ is called a pairing if it satisfies (i) and (ii) in Lemma 2.

Note that a pairing is different from a matching because it allows a node to be shared by multiple edges.

\section{A. Transmitter-Receiver Pairing}

From Lemma 2, it is clear that finding a power allocation can be decomposed into two steps. First, find a pairing, and then select the transmit power levels for the given pairing. Since there is a finite number of pairings, and one of them is optimal, it is easy to generate an optimal pairing with positive probability. One such algorithm is given by RANDPAIR (see Algorithm 2), which has $O(1)$ computation and communication complexity, and will find an optimal pairing with positive probability, as stated in the following lemma.

Lemma 3: Algorithm RAND-PAIR finds an optimal pairing with probability at least $(4 N)^{-N}$.

Proof: Consider an arbitrary pairing. Let $(a, b)$ denote one of the links in the pairing. The probability that this link is created (under RAND-PAIR) is $\frac{1}{2} \cdot \frac{1}{2} \cdot \frac{1}{|V(a)|} \geq \frac{1}{4 N}$. Since there can be at most $N$ links in a pairing, the probability that RAND-PAIR finds this pairing is at least $(4 N)^{-N}$. Hence, it will find an optimal pairing with probability at least $(4 N)^{-N}$.

Note that in the interference graph model, a new scheduling should be a max-weight matching (or independent set in 


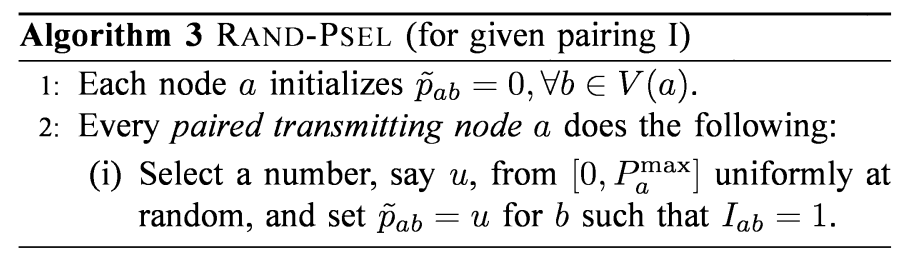

general) with positive probability. Because the max-weight matching is one of maximal matchings, and such a probability can be increased by performing multiple iterations until the obtained matching becomes maximal. However, in our case, maximal pairing may not be always optimal. Hence, performing multiple iterations does not necessarily enhance the probability of being optimal, and further it may not guarantee that the obtained pairing has a positive probability of being optimal.

\section{B. Power Level Selection}

Now what remains is to select a power level which together with RAND-PAIR satisfies $C 1$. Note that RAND-PAIR generates a pairing $I=\left[I_{a b}, b \in V(a), a \in V\right]$. Given this pairing, the problem (7) can be rewritten as:

$$
\begin{aligned}
& p^{*}(I)= \\
& \arg \max _{p \in F} \sum_{a \in V} \sum_{b: I_{a b}=1} q_{a b} \log \left(1+\frac{g_{a b} p_{a b}}{n_{b}+\sum_{1 \neq a} \sum_{j: I_{i j}=1} g_{i b} p_{i j}}\right) .
\end{aligned}
$$

Notice that the self-interference has been removed and the mutual interference has been simplified due to the constraints (i) and (ii) in Lemma 2. Since the pairing $I$ found by RANDPAIR has a positive probability of being optimal, the condition $C 1$ can be satisfied if a power level is selected such that it is within a factor of the objective in (8) with positive probability. To meet this requirement, Algorithm RAND-PSEL simply chooses power levels uniformly at random. In particular, each transmitting node a randomly selects its transmit power from the feasible region, i.e., $\left[0, P_{a}^{\max }\right]$. This random power selection meets the requirement as shown in the following lemma.

Lemma 4: Assume $P_{a}^{\max }=1, \forall a$. For any $\epsilon \in(0,1)$, Algorithm RAND-PSEL generates a power vector $\tilde{p}$ such that $\tilde{p} \in B\left(p^{*}(I), \epsilon\right)$ with probability at least $\left(\frac{\epsilon}{N}\right)^{N}$, where $B\left(p^{*}(I), \epsilon\right)=\left\{p \in F:\left\|p-p^{*}(I)\right\|_{2} \leq \epsilon\right\}$.

Proof: For given pairing $I$, each transmitting node $a$ will select its power level $\tilde{p}_{a}$ uniformly at random from $[0,1]$. Let $\operatorname{Tx}(I)$ be the set of transmitters under pairing $I$. Then, we have

$$
\begin{aligned}
& \operatorname{Pr}\left[\left\|\tilde{p}-p^{*}(I)\right\|_{2} \leq \epsilon\right] \\
& =\operatorname{Pr}\left[\sqrt{\sum_{a \in T x(I)}\left(\tilde{p}_{a}-p_{a}^{*}(I)\right)^{2}} \leq \epsilon\right] \\
& \geq \operatorname{Pr}\left[\left|\tilde{p}_{a}-p_{a}^{*}(I)\right| \leq \frac{\epsilon}{\sqrt{|T x(I)|}}, \forall a \in T x(I)\right] \\
& =\prod_{a \in T x(I)} \operatorname{Pr}\left[\left|\tilde{p}_{a}-p_{a}^{*}(I)\right| \leq \frac{\epsilon}{\sqrt{|T x(I)|}}\right] \\
& \geq \prod_{a \in T x(I)} \frac{\epsilon}{\sqrt{|T x(I)|}} \\
& \geq\left(\frac{\epsilon}{\sqrt{N}}\right)^{N}
\end{aligned}
$$

Note that this lemma can be easily extended to the case of general $P_{a}^{\max }$. Combining Lemmas 3 and 4, we can show that Condition $C 1$ can be satisfied by RAND-PAIR and RANDPSEL.

Theorem 2: Choosing a power allocation according to RAND-PAIR and RAND-PSEL satisfies $C 1$ with arbitrarily small $\gamma_{1}>0$ and $\delta_{1}>0$ (depending on $\gamma_{1}$ ).

Proof: Let $f(p)$ be the objective function in (8), and consider an arbitrary $\gamma_{1} \in(0,1)$. Due to the continuity of $f(p)$, there exists $\epsilon>0$ (depending on $\gamma_{1}$ such that $f(p) \geq\left(1-\gamma_{1}\right) f\left(p^{*}(I)\right)$ for any feasible $p$ such that $\left\|p-p^{*}(I)\right\| \leq \epsilon$. By Lemmas 3 and 4, the power allocation obtained through RAND-PAIR and RAND-PSEL achieves at least $\left(1-\gamma_{1}\right)$ fraction of optimal value of problem (7) with probability at least $\left(4 \epsilon\left(\gamma_{1}\right) N^{\frac{3}{2}}\right)^{-N}$, satisfying Condition $C 1$.

According to Theorems 1 and 2, the throughput loss due to the optimality loss $\left(\gamma_{1}\right)$ under our power allocation is negligible, as long as $\delta_{2} \ll \delta_{1}$. However, the probability $\delta_{1}$ under our power allocation policy is an extremely small number. Therefore, we will need a comparison algorithm that achieves very small or preferably zero $\delta_{2}$.

\section{GOSSIPING-BASED COMPARISON}

The goal of the COMPARE algorithm is to choose a power allocation $p(t)$ by selecting one of the two power allocations $p(t-1)$ and $\tilde{p}(t)$, so that Condition $C 2$ can be satisfied. Such a selection is easy in a centralized setting; namely, one can compare $q(t)^{T} r(p(t-1))$ and $q(t)^{T} r(\tilde{p}(t))$, and pick one having larger value. However, in multi-hop networks, centralized computation is prohibitive, hence we will use randomized gossiping [21] to implement COMPARE in a distributed manner.

Let $x_{b}^{\text {old }}$ and $x_{b}^{\text {new }}$ be the weighted (receiving) rates at node $b$ under the old power $p(t-1)$ and new power $\tilde{p}(t)$, respectively. Then, they can be expressed as $x_{b}^{\text {old }}=\sum_{a \in V} q_{a b}(t) r_{a b}(p(t-1))$ and $x_{b}^{\text {new }}=\sum_{a \in V} q_{a b}(t) r_{a b}(\tilde{p}(t))$. Let $x_{b}(0)=x_{b}^{\text {new }}-x_{b}^{\text {old }}$, i.e., $x_{b}(0)$ is the increment of weighted rate at node $b$ in case of changing from $p(t-1)$ to $\tilde{p}(t)$. Define $X$ as the network-wide increment of objective value, i.e., $X=\sum_{a \in V} x_{a}(0)$. Then, the COMPARE algorithm must choose the new power if $X>0$, and the old one if $X \leq 0$. This can also be accomplished using 


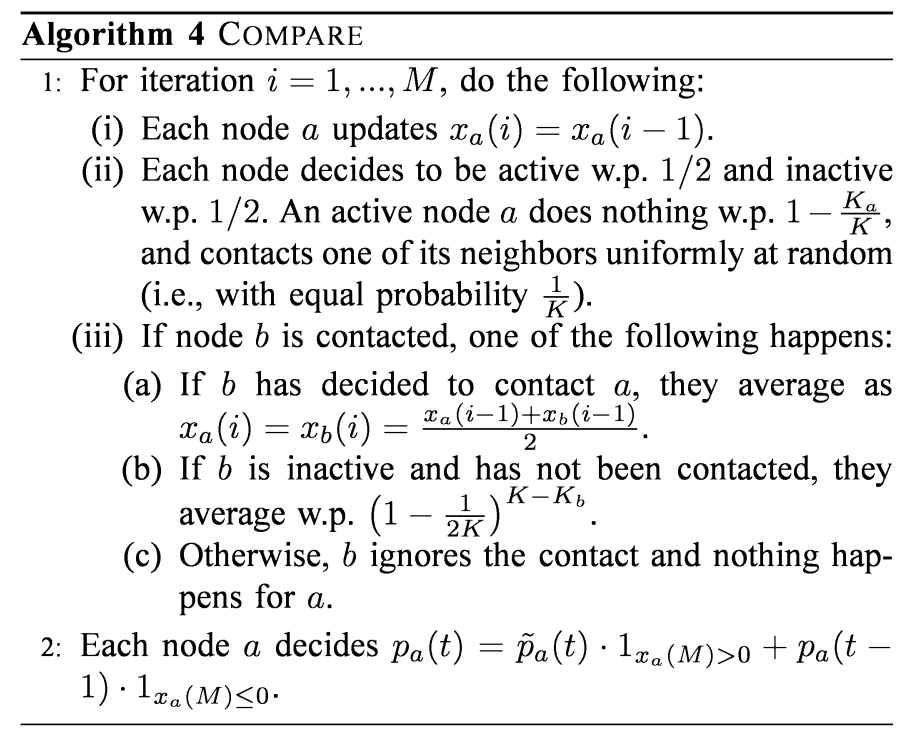

the average increment $\bar{X}=X / N$ instead of $X$, and hence if every node can compute an accurate estimate of $\bar{X}$, they can make a decision leading to $C 2$. A randomized gossiping algorithm is used to estimate $\bar{X}$.

Assume that each node $a$ has $x_{a}(0)$ (In fact, this value is computed in the training slot discussed in Section III-C). Let $K_{a}$ be the degree of node $a$, i.e., $K_{a}=|V(a)|$ and $K$ be the maximum node degree, i.e., $K=\max _{a \in V} K_{a}$. The comparison and decision is described in Algorithm COMPARE; where each node $a$ estimates the average $\bar{X}$ using gossiping, and after $M$ iterations, decides its transmit power vector $p_{a}(t)$ based on the estimation $x_{a}(M)$. The following is the restatement of the results on gossiping-based averaging in [21], [22].

Theorem 3: Under Algorithm COMPARE, there exists a finite number $M(N)$ such that for any $\epsilon, \delta>0, \operatorname{Pr}\left[x_{a}(m) \in\right.$ $((1 \pm \epsilon) \bar{X}), \forall a] \geq 1-\delta, \forall m \geq M(N)$, where $M(N)=$ $\Theta\left(-N^{3}(\log \epsilon+\log \delta)\right)$.

The number $M(N)$ is called $\epsilon$-convergence time. This result can be used to show that Algorithm COMPARE eventually satisfies $C 2$, in which case $M(N)=\Theta\left(-N^{3} \log \left(\gamma_{2} \delta_{2}\right)\right)$. Note that the $\epsilon$-convergence time is a conservative lower bound in that a unanimous decision satisfying $C 2$ can be made once all the nodes reach to the same sign of their estimates. This has led us to define a new concept of sign-wise convergence (or $s$-convergence).

Definition 3: A real-number vector $x$ is said to be uniform in sign (u.i.s.) if $x>0$ or $x \leq 0$ component wise.

Definition 4 (s-convergence time): For a sequence of vectors $\{x(m)\}$, the sign-wise convergence time $M^{s}(N)$ is defined by $M^{s}(N, \delta)=$ $\inf \{m \geq 0: \operatorname{Pr}[x(m)$ is u.i.s. $] \geq 1-\delta\}$.

Note that once a sequence $\{x(m)\}$ generated by Algorithm COMPARE becomes u.i.s., it will remain u.i.s. forever because the averaging operation between any nodes of the same sign does not change their signs. Moreover, after $M^{s}(N, \delta)$ iterations, the converged sign will be the same as $\bar{X}$ with probability $1-\delta$, because otherwise it will contradict to the previous result [21] that every node will get more accurate estimate of $\bar{X}$ as iteration proceeds. Therefore, for any $M \geq$ $M^{s}\left(N, \delta_{2}\right)$, Algorithm COMPARE will satisfy $C 2$ with $\gamma_{2}=0$. The following result is obvious.

Lemma 5: Assume $|\bar{X}| \geq \epsilon^{*}>0$, then for any $\epsilon<\epsilon^{*}$, $s$-convergence time is smaller than $\epsilon$-convergence time.

According to Lemma 5, the agreed decision satisfying $C 2$ can be reached faster than expected in the previous analysis (adopting $\epsilon$-convergence time). This will be verified through simulations in the next section.

\section{Simulation Results}

We generated a network topology by randomly placing $N$ nodes in a plane. For each link $(a, b)$, packets arrive according to a Poisson arrival process of rate $\lambda=0.5$, with the mean packet size of $2 \rho$. The offered load is thus $\rho$, and this parameter will be changed to examine the algorithm performance. Let $d_{a b}$ be the distance between nodes $a$ and $b$. The channel gain $g_{a b}$ is fixed to $1 /\left(1+d_{a b}^{4}\right)$ if $a \neq b$, and as assumed in Section II, $g_{a b}=\infty$ if $a=b$. The noise power and the maximum transmit power are fixed as $n_{a}=0.01$ and $P_{a}^{\max }=1$ for every node $a$.

Figure 2(a) compares $\epsilon$-convergence time and sign-wise convergence time of the gossiping-based averaging in Algorithm COMPARE. The $\epsilon$-convergence time increases quadratically in number of nodes, whereas the $s$-convergence time increases linearly. Hence, the gossiping-based decision can satisfy $C 2$ with much less iterations than expected in the previous analysis. Figure 2(b) plots the stability performance of gossiping-based comparison (Algorithms RAND-PAIR, RANDPSEL and COMPARE; denoted by gossip) and centralized comparison (Algorithms RAND-PAIR, RAND-PSEL with centralized comparison; denoted by centralized comp.). The centralized comparison satisfies $C 2$ with $\gamma_{2}=0$ and $\delta_{2}=0$, and hence by Theorem 1, it achieves nearly $100 \%$ throughput. As the number of iterations ( $M$ in Algorithm 4) increases, the performance of gossiping approaches that of centralized comparison. This implies that our distributed power control scheme can achieve maximum throughput.

\section{CONCLUSION}

We considered the problem of achieving maximum throughput under SINR-based rate model in multi-hop wireless networks. Typically, this requires repeatedly solving an optimal power allocation problem by taking into account channel conditions and queue backlog information. However, finding such a power allocation for every time slot is impractical due to not only the difficulty of the problem but also the need for distributed operation. In order to circumvent this difficulty, we use a randomization approach that has been successfully applied to graph-based interference model. To that end, we developed a new optimality condition for random power allocation that enables the application of the randomization framework to the throughput-optimal power allocation problem. We also developed a randomized power allocation that satisfies the new 


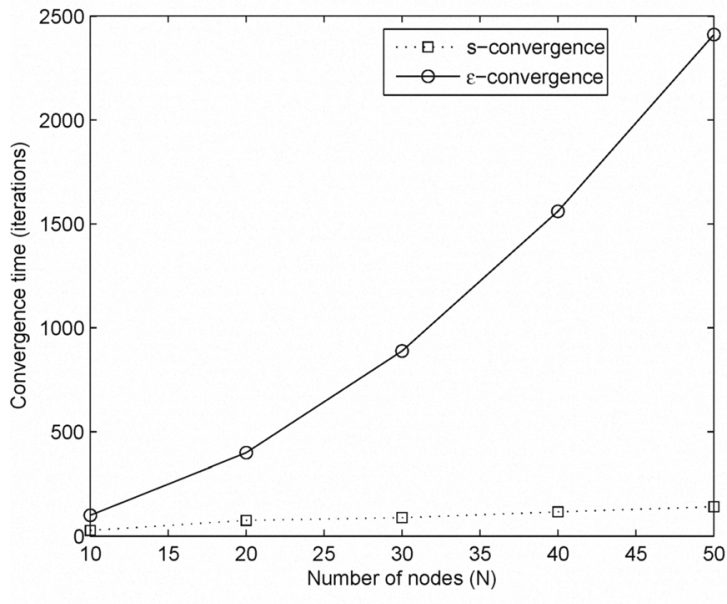

(a) Convergence time: $\epsilon=0.001$

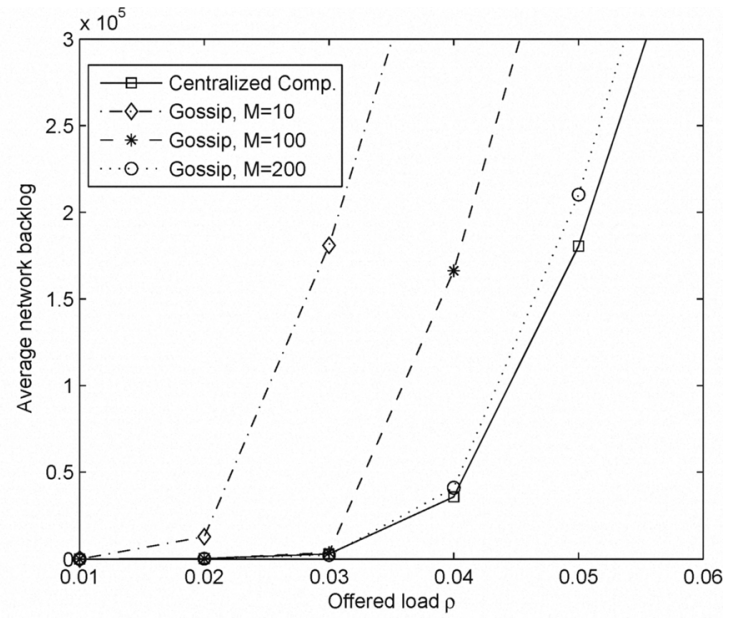

(b) Network backlog vs. offered load ( $N=16$

Fig. 2. Convergence time and stability performance

optimality condition, and a distributed gossip-based comparison mechanism that achieves $100 \%$ throughput, together with the randomized power allocation. Future directions include extending this result to the network with multi-hop traffic and multiple channels.

\section{APPENDIX A}

PROOF OF THEOREM 1

Consider the following Lyapunov function

$$
L(q(t)):=\sum_{(a, b) \in E} q_{a b}(t)^{2} .
$$

Define a one-step Lyapunov drift as follows:

$$
L(q(t+1))-L(q(t)):=\sum_{(a, b) \in E}\left[q_{a b}(t+1)^{2}-q_{a b}(t)^{2}\right] .
$$

For notational convenience, we will omit time index $t$ when $q$ refers to $q(t)$ ). Using the queue evolution equation (2), we have

$$
\begin{aligned}
q_{a b}(t+1)^{2} & \leq\left(q_{a b}-r_{a b}\right)^{2}+A_{a b}^{2}+2 A_{a b} \max \left[0, q_{a b}-r_{a b}\right] \\
& \leq\left(q_{a b}-r_{a b}\right)^{2}+A_{a b}^{2}+2 A_{a b} q_{a b} \\
& =q_{a b}^{2}+2 q_{a b}\left(A_{a b}-r_{a b}\right)+A_{a b}^{2}+r_{a b}^{2} .
\end{aligned}
$$

Hence, the one-step Lyapunov drift can be bounded as we refer to a value of any variable at this time instant (e.g.,

Therefore, the expected conditional $T$-step Lyapunov drift can be bounded as

$$
\begin{aligned}
\Delta_{T}(t) & =E\{L(q(t+T))-L(q(t) \mid q(t))\} \\
\leq 2 \sum_{\tau=0}^{T-1} \sum_{(a, b) \in E} E\left\{q _ { a b } ( t + \tau ) \left(A_{a b}(t+\tau)\right.\right. & \left.\left.-r_{a b}(t+\tau)\right) \mid q(t)\right\} \\
+ & \sum_{\tau=0}^{T-1} \sum_{(a, b) \in E} E\left\{A_{a b}(t+\tau)^{2}+r_{a b}(t+\tau)^{2} \mid q(t)\right\} .
\end{aligned}
$$

We observe that the link rate can be upper-bounded as

$$
r_{a b} \leq \log \left(1+\frac{g_{a b} P_{a}^{\max }}{n_{b}}\right)=r_{a b}^{\max }
$$

where the equality holds when link $(a, b)$ uses the maximum power and there is no interference from other nodes in the network. Therefore, under the assumption that the link arrival processes have bounded second moments, the term in (15) is bounded by a finite number $B_{1}$. Also, because the arrival processes are independent of the queue length processes in any time slot $t+\tau$, we have

$\Delta_{T}(t) \leq 2 \sum_{\tau=0}^{T-1} E\left\{q_{a b}(t+\tau)\left(\lambda_{a b}-r_{a b}(t+\tau)\right) \mid q(t)\right\}+B_{1}$. Now, let us define

$$
\Psi(t):=q(t)^{T} r^{*}(t)-q(t)^{T} r(t)
$$

Using this result for $t, t+1, \cdots, t+T-1$ and sum both sides together, we can obtain the following

$$
\begin{aligned}
& L(q(t+T))-L(q(t)) \\
& \leq 2 \sum_{\tau=0}^{T-1} \sum_{(a, b) \in E} q_{a b}(t+\tau)\left(A_{a b}(t+\tau)-r_{a b}(t+\tau)\right) \\
& \quad+\sum_{\tau=0}^{T-1} \sum_{(a, b) \in E}\left\{A_{a b}(t+\tau)^{2}+r_{a b}(t+\tau)^{2}\right\} .
\end{aligned}
$$

where $r^{*}(t)$ is the optimal rate which corresponds to the optimal power allocation given the queue length vector $q(t)$ at time $t$ (i.e., it achieves the maximum weight). Then, we have

$$
\begin{array}{r}
\Delta_{T}(t) \leq 2 \sum_{\tau=0}^{T-1} E\left\{q(t+\tau)^{T} \lambda-q(t+\tau)^{T} r^{*}(t+\tau) \mid q(t)\right\} \\
+B_{1}+2 \sum_{\tau=0}^{T-1} E\{\Psi(t+\tau) \mid q(t)\}
\end{array}
$$


Define the following quantities

$$
\begin{aligned}
& Z_{1}:=\inf _{\tau \geq 0}\left\{q(t+\tau)^{T} r(t+\tau) \geq \gamma_{1} q(t+\tau)^{T} r^{*}(t+\tau)\right\}\left(20 \text { fails at time } t+Z_{1}+\tau\right\}-t \\
& Z_{2}:=\inf _{\tau \geq 0}\{\mathrm{C} 2 \text { fals }
\end{aligned}
$$

Now, we try to upper-bound the last term in (19). First, note that because both link rate and the amount of arriving traffic in any time slot are bounded (see (16)), we have for any $0 \leq$ $\tau \leq T$

$$
\begin{aligned}
\Psi(t+\tau) & \leq q(t+\tau)^{T} r^{*}(t+\tau) \\
& \leq q(t)^{T} r^{*}(t)+B_{2}
\end{aligned}
$$

where $B_{2}=T N A_{\max } R_{\max }$ and $R_{\max }=\max _{\{(a, b) \in E\}} r_{a b}^{\max }$ and $A_{\max }$ is the maximum amount of traffic arriving in one time slot at any links. Also, under conditions $\mathrm{C} 1$ and $\mathrm{C} 2$, we have for $Z_{1} \leq \tau \leq \min \left\{T, Z_{2}\right\}$

$$
\begin{aligned}
W(t+\tau) \geq\left(1-\gamma_{1}\right)\left(1-\gamma_{2}\right) W^{*}\left(t+Z_{1}\right) & \\
& -\left(\tau-Z_{1}\right) N R_{\max }^{2} \\
\geq\left(1-\gamma_{1}\right)\left(1-\gamma_{2}\right) W^{*}(t)- & T N R_{\max }^{2} .
\end{aligned}
$$

where $W^{*}(t)=q(t)^{T} r^{*}(t)$ and $W(t)=q(t)^{T} r(t)$. Using this result, we have for $Z_{1} \leq \tau \leq \min \left\{T, Z_{2}\right\}$

$$
\begin{aligned}
\Psi(t+\tau)= & W^{*}(t+\tau)-W(t+\tau) \\
\leq & W^{*}(t)+T N A_{\max } R_{\max } \\
& -\left(1-\gamma_{1}\right)\left(1-\gamma_{2}\right) W^{*}(t)+T N R_{\max }^{2} \\
= & {\left[\gamma_{1}+\left(1-\gamma_{1}\right) \gamma_{2}\right] W^{*}(t)+B_{3} }
\end{aligned}
$$

where $B_{3}=T N A_{\max } R_{\max }+T N R_{\max }^{2}$ is a finite number. Using (23) and (25), we have

$$
\begin{aligned}
\sum_{\tau=0}^{T-1} \Psi(t+\tau) \leq( & \left.W^{*}(t)+B_{2}\right)\left(\min \left\{T, Z_{1}\right\}+\hat{T}\right) \\
+ & \left\{\left[\gamma_{1}+\left(1-\gamma_{1}\right) \gamma_{2}\right] W^{*}(t)+B_{3}\right\} T \\
= & W^{*}(t)\left(\min \left\{T, Z_{1}\right\}+\hat{T}\right) \\
+ & {\left[\gamma_{1}+\left(1-\gamma_{1}\right) \gamma_{2}\right] W^{*}(t) T+B_{4} }
\end{aligned}
$$

where $\hat{T}=T-\min \left\{T, Z_{2}\right\}$ and $B_{4}$ is a finite number. Now, we have

$E\left\{\min \left\{T, Z_{1}\right\}\right\} \leq E\left\{\min \left\{Z_{1}\right\}\right\} \leq \frac{1}{\delta_{1}}$

$E\{\hat{T}\}=E\left\{T-\min \left\{T, Z_{2}\right\}\right\} \leq T \operatorname{Pr}\left[T \geq Z_{2}\right]=\delta_{2} T^{2}$

where in (28) we have used the fact that $\operatorname{Pr}\left[T \geq Z_{2}\right] \leq \delta_{2} T$. Substitute (27) and (28) into (26), we have

$$
\begin{aligned}
\sum_{\tau=0}^{T-1} \Psi(t+\tau) \leq T\left(\gamma_{1}+(1-\right. & \left.\gamma_{1}\right) \gamma_{2}+\frac{1}{\delta_{1} T} \\
& \left.+\delta_{2} T\right) W^{*}(t)+B_{4} .
\end{aligned}
$$

Now, we upper-bound the first term in (18). First, recall that link rates are upper-bounded by $R_{\max }$, and we have assumed the amount of arriving traffic in one time slot is bounded. Also, for any arrival rate lies within the capacity region, we have $\lambda_{a b} \leq R_{\max }$. Therefore, we have

$$
\begin{aligned}
& \sum_{\tau=0}^{T-1}\left\{q(t+\tau)^{T} \lambda-q(t+\tau)^{T} r^{*}(t+\tau)\right\} \\
& \leq T\left[q(t)^{T} \lambda-q(t)^{T} r^{*}(t)\right]+B_{5}
\end{aligned}
$$

where $B_{5}$ is a finite number. Now, consider any arrival rate vector $\lambda$ which lies strictly inside the $\rho$-scaled capacity region (i.e., inside $\rho \Lambda$ ). Then, there exists a feasible power allocation vector $p$ for any backlog vector $q$ such that

$$
q^{T} \lambda \leq \rho q^{T} r(p) \leq \rho q^{T} r^{*}
$$

where $r^{*}$ is the optimal rate which achieves the maximum weight (i.e., $q^{T} r^{*}=\max _{p \in F} q^{T} r(p)$ ). Suppose $\lambda$ lies strictly inside the $\rho$-scaled capacity region, then there exists a vector $\epsilon 1$ such that $\lambda+\epsilon 1$ also lies strictly inside the $\rho$-scaled capacity region where 1 is a vector of all ones. That means we have the following

$$
q^{T}(\lambda+\epsilon \mathbf{1}) \leq \rho q^{T} r^{*}
$$

or

$$
q^{T} \lambda-q^{T} r^{*} \leq-(1-\rho) q^{T} r^{*}-\epsilon q^{T} \mathbf{1} .
$$

Using this result to (30), for any arrival vector $\lambda$ strictly inside the $\rho$-scaled capacity region we have

$$
\begin{aligned}
& \sum_{\tau=0}^{T-1}\left\{q(t+\tau)^{T} \lambda-q(t+\tau)^{T} r^{*}(t+\tau)\right\} \\
& \quad \leq T\left[-(1-\rho) q(t)^{T} r(t)^{*}-\epsilon q(t)^{T} \mathbf{1}\right]+B_{5} .
\end{aligned}
$$

Using (29) and (34), the expected conditional $T$-step Lyapunov drift in (18)-(19) can be bounded as

$$
\begin{array}{r}
\Delta_{T}(t) \leq-2 T\left\{1-\rho-\gamma_{1}-\left(1-\gamma_{1}\right) \gamma_{2}-\frac{1}{\delta_{1} T}-\delta_{2} T\right\} W^{*}(t) \\
-2 \epsilon T q(t)^{T} \mathbf{1}+B_{6}
\end{array}
$$

where $B_{6}=B_{1}+2 B_{5}+2 B_{4}$ is a finite number. Now, if we choose $T=\sqrt{1 /\left(\delta_{1} \delta_{2}\right)}$, we have

$$
\begin{array}{r}
\Delta_{T}(t) \leq-T\left\{1-\rho-\gamma_{1}-\left(1-\gamma_{1}\right) \gamma_{2}-2 \sqrt{\frac{\delta_{2}}{\delta_{1}}}\right\} W^{*}(t) \\
-2 \epsilon T q(t)^{T} 1+B_{6} .
\end{array}
$$

Therefore, for any $\rho<1-\gamma_{1}-\left(1-\gamma_{1}\right) \gamma_{2}-2 \sqrt{\frac{\delta_{2}}{\delta_{1}}}, \Delta_{T}(t)$ becomes negative when $q(t)^{T} \mathbf{1}$ is large enough. Hence, the network queue is stable.

\section{REFERENCES}

[1] X. Lin, N. B. Shroff, and R. Srikant, "A tutorial on cross-layer optimization in wireless networks," IEEE J. Select. Areas Commun., vol. 24 no. 8, pp. 1452-1463, Aug. 2006.

[2] G. Sharma, N. B. Shroff, and R. R. Mazumdar, "On the complexity of scheduling in wireless networks," in $A C M$ MOBICOM, Los Angeles, CA, Sept. 2006.

[3] E. Modiano, D. Shah, and G. Zussman, "Maximizing throughput in wireless networks via gossiping," in ACM SIGMETRICS/Performance, Saint-Malo, France, June 2006. 
[4] A. Eryilmaz, A. Ozdaglar, and E. Modiano, "Polynomial complexity algorithms for full utilization of multi-hop wireless networks," in IEEE INFOCOM, Anchorage, AK, May 2007.

[5] S. Sanghavi, L. Bui, and R. Srikant, "Distributed link scheduling with constant overhead," in ACM SIGMETRICS, San Diego, CA, June 2007.

[6] A. Gupta, X. Lin, and R. Srikant, "Low-complexity distributed scheduling algorithms for wireless networks," in IEEE INFOCOM, Anchorage, AK, May 2007.

[7] R. Cruz and A. Santhanam, "Optimal routing, link scheduling and power control in multihop wireless networks," in IEEE INFOCOM, San Francisco, CA, June 2006.

[8] T. ElBatt and A. Ephremides, "Joint scheduling and power control for wireless ad hoc networks," IEEE Trans. Wireless Commun., vol. 3, no. 1, pp. 74-85, Jan. 2004.

[9] M. Chiang, "Balancing transport and physical layers in wireless multihop networks: Jointly optimal congestion control and power control," IEEE J. Select. Areas Commun., vol. 23, no. 1, pp. 104-116, Jan. 2005.

[10] B. Radunovic and J. Le Boudec, "Optimal power control, scheduling, and routing in UWB networks," IEEE J. Select. Areas Commun., vol. 22, no. 7, pp. 1252-1270, Sep. 2004

[11] S. A. Borbash and A. Ephremides, "Wireless link scheduling with power control and sinr constraints," IEEE Trans. Inform. Theory, vol. 52, no. 11 , pp. 5106-5111, Nov. 2006

[12] L. Tassiulas and A. Ephremides, "Stability properties of constrained queueing systems and scheduling policies for maximum throughput in multihop radio networks," IEEE Trans. Automat. Contr., vol. 37, no. 12 pp. 1936-1948, Dec. 1992.

[13] M. J. Neely, E. Modiano, and C. E. Rohrs, "Power allocation and routing in multibeam satellites with time-varying channels," IEEE/ACM Trans. Networking, vol. 11, no. 1, pp. 138-152, Feb. 2003.

[14] M. Neely, E. Modiano, and C. Rohrs, "Dynamic power allocation and routing for time varying wireless networks," IEEE J. Select. Areas Commun., vol. 23, no. 1, pp. 89-103, Jan. 2005

[15] M. Neely and E. Modiano, "Fairness and optimal stochastic control for heterogeneous networks," in IEEE INFOCOM, Miami, FL, Mar. 2005.

[16] X. Lin and N. Shroff, "The impact of imperfect scheduling on cross-layer congestion control in wireless networks," IEEE/ACM Trans. Networking, vol. 14 , no. 2 , pp. 302-315, Apr. 2006.

[17] P. Charporkar, K. Kar, and S. Sarkar, "Throughput guarantees through maximal scheduling in wireless networks," IEEE Trans. Inform. Theory, vol. 54, no. 2, pp. 572-594, Feb. 2008.

[18] G. Zussman, A. Brzezinski, and E. Modiano, "Multihop local pooling for distributed throughput maximization in wireless networks," in IEEE INFOCOM, Phoenix, AZ, April 2008.

[19] C. Joo, X. Lin, and N. B. Shroff, "Understanding the capacity region of the greedy maximal scheduling algorithm in multi-hop wireless networks," in IEEE INFOCOM, Phoenix, AZ, April 2008.

[20] L. Tassiulas, "Linear complexity algorithms for maximum throughput in radio networks and input queued switches," in IEEE INFOCOM, San Francisco, CA, March 1998.

[21] S. Boyd and et al., "Randomized gossip algorithms," IEEE Trans. Inform. Theory, vol. 52, no. 6, pp. 2508-2530, June 2006.

[22] A. Olshevsky and J. N. Tsitsiklis, "Convergence speed in distributed consensus and averaging," SIAM Journal on Control and Optimization, vol. 48, no. 1, pp. 33-55, 2009. 